\title{
Review and perspective on 3D Gesture Recognition Technologies for Automotive Industry
}

\author{
Piotr Reczek 1) 2) Andrzej Wetula ${ }^{12)}$ Andrzej Młyniec ${ }^{1)}$ \\ 1) AGH University of Science and Technology \\ Mickiewicza 30, 30-059 Kraków, Poland (website:/www.agh.edu.pl) \\ 2) Merit Poland Sp. z o. o. \\ Polode 60, 30-394Krakow, Poland
}

Received on July 5,2021

\begin{abstract}
The paper reviews 3D Gesture Recognition technologies, that can be potentially used in future automotive applications. The purpose of the article is to find the best 3D gesture recognition technology for mass scale adaptation in future vehicles. Described gesture recognition technologies are divided into six categories: Vision Systems, Wearable sensors, Infrared, Electric field, Radar and Ultrasonic Waves. The article identifies principles of operation for each of chosen methods, compares advantages and disadvantages of the technologies.
\end{abstract}

KEY WORDS: Gestures recognition, Automotive, Human Machine Interface (E2)

\section{Introduction}

Research in the field of Human Machine Interfaces is extensive in the last years. 3D gesture recognition systems are one of the recent ideas for interaction between a human and a vehicle. Interfaces of this type are present in premium vehicles, but they are not available in an medium-class car, due to high unit cost. Therefore, popularization of $3 \mathrm{D}$ gesture recognition systems requires solutions that are relatively inexpensive in an implementation.

This article reviews gesture recognition methods, that can be used in automotive industry. The methods were divided by technologies. Each paragraph shows one technology analysis and indicates the research gaps that stops adaptation into car industry on a mass scale.

\section{3D GESTURE TECHNOLOGIES REVIEW}

\subsection{Vision Systems}

In the automotive industry first 3D Gesture Recognition system was introduced by BMW (1). Research about the BMW system began at least 20 years ago, but it has been introduced 15 years later (2). Those numbers show the complexity of the system and how long it takes to introduce innovative technologies in automotive industry. Very important topic in case of gesture recognition is operating area range. In BMW solution (1) camera is located more less $50 \mathrm{~cm}$ above the user hand. It is beneficial, because of large space, where gestures are detectable. On the other hand, this system do not provide subtle gestures detection (gesture performed only with the fingers). To make it possible, higher resolution camera shall be installed. Other possibility is putting camera closer to the user's hand eg. in front of main central display. Then gestures must be performed closed to the camera, but variety of the gestures will be greater.

\subsection{Electric field}

Nowadays, electric field sensors are used in majority of touch screens and touch pads. Their size varies depending on application - from invisible dots on the screens to few centimeters wide polygons. 3D gesture recognition systems based on electric field sensors use the same physical effect.

Electric field sensors are better known as capacitive sensors. Generally, in a model example, we have two electrodes transmitting and receiving. At the transmitting electrode variable voltage is applied. The result is generation of an electromagnetic field between electrodes. Receiving electrode collects electric charge. The amount of charge is proportional to capacitance between the electrodes. When the user hand is present in the area between electrodes, a surrounding electrical field is disturbed. In the effect, some charge is transferred into the user hand, while the rest flows into the receiving electrode. As a result, receiving electrode gains lower charge than it does without the hand present. This description concerns detection based on mutual capacitive sensors. Another method of sensing is self-capacitance, but it is not used for 3D gestures recognition. Self-capacitance method can be considered as merging transmitting and receiving electrodes into one. The same actions like for mutual method are needed. they are preformed sequentially on single electrode. In case of gesture recognition important aspect is distance between electrodes and object. Mutual method wins in this category.

The sensors are used in following systems: "hands free" trunk opening, hand proximity in door handle (3), foot gesture recognition system (4). However, these systems detect movement of whole limbs. Today car systems require more complex input. In response to this need, engineers and researchers have been working on providing systems that are able to detect more subtle gestures. For example, Microchip company introduced automotive qualified technology called Gestic (5). It includes dedicated chipsets and 
evaluation software. Gesture recognition algorithm is based on Hidden Markov Model and it is implemented inside the chip. The datasheet indicates gestures recognized by the device: Swipes (up,down, right, left), Circle Gestures ( single or multiple clockwise and anticlockwise), Approach and Taps . Such solutions shorten time from an idea to an application. We can find Gestic in Jaguar XF Sportbrake from 2018 (6). It detects gestures that open and close roof window blind. User opens the blind with swipe back gesture, closes with swipe forward. System is mounted in the roof module, near a rear-view mirror. Active area, where gestures are detected, is located a few centimeters below the module.

Some publications describe capacitive hand gesture recognition systems solutions based on custom systems (7),(8). They have specially designed electrodes, and signal processing is done with dedicated electronics.

Mentioned research and products show that we can expect 3D gesture recognition systems in the near future.

\subsection{Infrared}

All the infrared gesture recognition systems use at least one infrared light emitting diode (LED). It emits the light in the range between $730-950 \mathrm{~nm}$. The light is reflected from an object and falls on a photosensitive electronic component - photodiodes or phototransistors. These elements provide output signal proportional to an intensity of a reflected light.

The simplest solution is to use only one receiving element, that reports intensity of a light reflected in a single point. Such a system can be used only for object proximity detection. For more advanced gesture sensors, more detecting points are needed. We have found many papers describing systems that use this technology for 3D gesture recognition systems. They have various sensors location solutions - from three receivers placed in a triangular pattern (9) to rectangle matrices, that can be considered as primitive cameras (10). In this case it is hard to set strict border between matrices of infrared sensors and simple infrared cameras. However, all solutions used Machine Learning Methods for interpretation of the collected data.

Received light intensity alone does not give much information about moving object. Fortunately, all things happen in the time domain. Thanks to that we can obtain light intensity vs time diagrams. Those diagrams contain much more useful features that can be valuable input to Machine Learning Algorithms. For example: time and angle of the edges, amplitude, variations, slope etc. (9). In effect, much more data is available from these infrared-based systems than it seems at the first look.

In the automotive industry, first known application of the infrared objects sensing in a mass scale, is proximity sensing in "Cadillac User Experience System". Two infrared sensors below the screen detect when user hand approaches the central panel screen (11). First 3D gesture recognition system based on infrared sensor were presented by Volkswagen (12),(13),(14). It enabled controlling the content of the multimedia system in a car with gestures such as simple swipes. Choosing the options is done with touch of the screen.

\subsection{Radar}

Radar technology is known for more than 100 years. For all that time it was used for detecting objects like ships and planes. In the last decade radar technology become to be implemented in automotive industry. Safe system captures scanned data in front of the car from the sensor, judges the situation and takes decision about breaking the car when a pedestrian is crossing the car's route. Similar application can be found in autonomous driving systems. Data provided by radars is crucial in this case - system must be aware of the environment around the whole car. Thanks to this data, the car knows location of the obstacles.

Due to the fact that the technology is already used in automotive, the chances for using it in 3D gesture recognition system are higher. Moreover, we have found two potential hardware sets that can be applied into this purpose: $\mathrm{mm}$-Wave sensor (Texas Instruments) (15) and Soli (Google) (16) . Both are operating with high frequency electromagnetic waves $(60 \mathrm{GHz})$, that corresponds with wavelength of $5 \mathrm{~mm}$. Wavelength shall be similar dimensions to the detected object, as this frequency range allows for detection of fingers and their movements.

We have found two articles, describing application of the abovementioned platforms and applications of the available hardware.

First one is mmWave sensor (17), that was placed in two locations on the car cockpit. Authors used Random Forest Classification method for the gesture detection algorithm. They analyzed six possible gestures: "Low wiggle", "Turn over", "High grab", "Swipe", "Large circle", Small Circle". These gestures are similarly complex as the ones detected with previously described technologies.

Second paper (18) - about the Soli - describes applying many Machine Learning methods for gesture detecting algorithm. The best results were achieved with "End-to-end trained combination of deep convolution and recurrent neural network". Recognition rate was $87 \%$. It could seem not so high, but an algorithm was able to recognize eleven kinds of gestures. The feature that differs soli from the other technologies is its resolution. It enabled gestures that need only two fingers e.g. Finger slide, Finger Rub. This fact can decide about placing this technology into new, unexpected and relatively small localization like wiper switch lever or single button surface.

\subsection{Ultrasonic Waves}

Next interesting candidate-technology for 3D gesture recognition is ultrasonic waves. This common method of sensing objects has found applications in USG and non-destructive material testing in metal engineering. In automotive industry, most of the new cars has ultrasonic parking sensors and ultrasonic intrusion detection sensors.

Radars operate when the ultrasonic wave is emitted by transmitter until encounters an object that reflects the waves into a receiver, similar to speaker and a microphone (19). The paper describes usage of speaker and microphone in a typical smart phone or tablet for $3 \mathrm{D}$ gestures recognition. Moreover, detecting algorithm was deployed on the mobile device.

Similarly to other technologies, machine learning methods are used for detection algorithms (20). All found examples used Doppler effect for detecting the movement of the hand. 
Other interesting example is the paper (21), that compares ultrasonic sensors with infrared sensors. Both types of sensors are located in the same places. According the results, infrared sensors are highly dependent on light conditions. Indoor tests results were few times better than outdoor. In case of ultrasonics sensors, no difference in accuracy were observed. The author concludes that: "...the light dependent method is more convenient for indoor application. But, for outdoor use the ultrasonic method is more convenient". Infrared sensors were many times cheaper, that is the reason that author suggest using them for indoor applications.

\subsection{Wearable sensors}

In the article context I want to narrow the topic to sensors integrated into a bracelet worn on a wrist. Nowadays, many people own smartwatches or personal coach devices on their hands. These devices can be controlled by simple gestures of a hand. But what if they could be a source of data for $3 \mathrm{D}$ gesture system in a car? Examples mentioned in this paragraph indicate that technically it is possible. The only doubt is the customers reception.

The first paper proposes accelerometers as a source of data (22). They are mounted in a thin bracelet and enable determining movement of the hand in the coordinate system in a car. Very big advantage of this technology is robustness to environmental conditions. Possibility of making gestures in the area inside and even outside the car is another remarkable point. Additional power supply for the bracelet is first disadvantage. Another is communication between bracelet and a car. It must be reliable due to required fast reaction on gestures. Those points are undoubtedly first challenges that must be overcome. For gesture detection machine learning algorithms were used, namely a Hidden Markov model. This method base on the relations between probabilities of the states and outputs. Basing on the outputs and transitions probabilities, probability of all possible states (gestures in our case) are calculated. Gesture which probability is the highest, is taken as the actual state.

In the second paper (23), authors describe another approach to the problem. They presented a bracelet, that consists of two subsystems: accelerometers and an infrared receiver. They also included a microcontroller on a bracelet. Additionally, system involved a main board with an FPGA circuit. The main task of the bracelet microcontroller is to collect data from accelerometers and infrared sensor, and send them into main board with FPGA, that has implemented Artificial Neurons Network algorithm for gesture recognition. In my opinion, the most surprising part is the Iinfrared sensor on the bracelet is the solution that I have not found anywhere else in the literature. It receives infrared signals send by beacons located in the active area of gesture recognition. This is another example, that shows we can expect merging of technologies in the future gesture recognition systems.

Last, but probably the most unique example (24), is the paper describing prototype of bracelet with two arrays of force sensitive resistors. Sensors are located around the bracelet adjoining to human skin. When the user moves a wrist or fingers, the sensors are pressed on by muscles. System was able to detect 24 gestures ( 18 by fingers, and 6 by a wrist). According to the author, the maximum achieve classification accuracy was $97.40 \%$ using a kNN algorithm.

\section{Conclusion}

During my research we have found dozen approaches to the 3D gesture recognition systems. First gesture recognition systems are already implemented in cars available on the market. Although they are very impressive, people always seek for further innovations.

As a summary we present two tables systematizing gathered data. In Table 1. we compared technologies in three categories: technology majority, resolution of the gestures and cost. Majority is expressed by Technology readiness level, that is standard created in that purpose. Technologies that have already implemented in cars got the highest - level 9. My classification criteria for gesture resolution is binary. We created two categories: detectable gestures performed with hand only, or with hand and fingers. We have found examples that four technologies were used in solutions that were able to recognize gestures done with fingers. Third category assumes mainly cost of electrical components.

Table 1. Technologies comparison

\begin{tabular}{lccc}
\hline Technology & $\begin{array}{c}\text { Technology } \\
\text { readiness level }\end{array}$ & $\begin{array}{c}\text { Gestures } \\
\text { with } \\
\text { fingers* }\end{array}$ & $\begin{array}{c}\text { Estimated } \\
\text { System cost }\end{array}$ \\
\hline $\begin{array}{l}\text { Vision } \\
\text { Systems }\end{array}$ & TRL 9 & yes & +++ \\
Electric field & TRL 9 & no & + \\
Infrared & TRL 9 & no & + \\
Radar & TRL 5 & yes & ++ \\
$\begin{array}{l}\text { Ultrasonic } \\
\begin{array}{l}\text { Waves } \\
\text { Wearable } \\
\text { sensors }\end{array}\end{array}$ & TRL 4 & yes & + \\
\hline
\end{tabular}

*Gestures with fingers - User use only his fingers for gesture performing. Wrist is quasi-static

The Table 2. expresses Advantages and limitations for each of technologies.

Table 2. Technologies advantages and disadvantages

\begin{tabular}{lll}
\hline Technology & \multicolumn{1}{c}{ Advantages } & \multicolumn{1}{c}{ Disadvantages } \\
\hline $\begin{array}{l}\text { Vision } \\
\text { Systems }\end{array}$ & Precise data & Complex system \\
& $\begin{array}{l}\text { Multiplicity of } \\
\text { gestures }\end{array}$ & Privacy issues \\
& Gigabytes of data \\
& $\begin{array}{l}\text { Well known } \\
\text { algorithms for vision } \\
\text { processing }\end{array}$ & \\
\hline $\begin{array}{l}\text { Electric } \\
\text { field }\end{array}$ & $\begin{array}{l}\text { Simple hardware }- \\
\text { only design of } \\
\text { electrodes is needed }\end{array}$ & $\begin{array}{l}\text { Too low resolution } \\
\text { for "Fingers } \\
\text { gestures" }\end{array}$ \\
& $\begin{array}{l}\text { Touch and 3D } \\
\text { gestures in the same } \\
\text { system }\end{array}$ & $\begin{array}{l}\text { Limited gesture } \\
\text { detection area }\end{array}$ \\
& $\begin{array}{l}\text { System invisible to } \\
\text { the driver }\end{array}$ & \\
\hline
\end{tabular}




\begin{tabular}{|c|c|c|}
\hline Infrared & $\begin{array}{l}\text { Simple hardware - } \\
\text { basic optoelectronics } \\
\text { component needed }\end{array}$ & $\begin{array}{l}\text { Disturbed by sunlight } \\
\text { Complex gestures } \\
\text { require high number } \\
\text { of components } \\
\text { Limited gesture } \\
\text { detection area }\end{array}$ \\
\hline & Easy to scale up/down & \\
\hline Radar & $\begin{array}{l}\text { System invisible to } \\
\text { the user } \\
\text { Small gestures } \\
\text { available }\end{array}$ & $\begin{array}{l}\text { Challenging } \\
\text { development of } \\
\text { antennas } \\
\text { Complicated signal } \\
\text { processing }\end{array}$ \\
\hline $\begin{array}{l}\text { Ultrasonic } \\
\text { Waves }\end{array}$ & $\begin{array}{l}\text { Wide technology } \\
\text { usage in automotive } \\
\text { Large operating area }\end{array}$ & $\begin{array}{l}\text { Cannot be completely } \\
\text { invisible - } \\
\text { transmitters } \\
\text { cannot be fully } \\
\text { covered }\end{array}$ \\
\hline $\begin{array}{l}\text { Wearable } \\
\text { sensors }\end{array}$ & $\begin{array}{l}\text { Bracelet can be } \\
\text { merged with watch } \\
\text { Gestures possible } \\
\text { outside the car } \\
\text { Many possible } \\
\text { gestures }\end{array}$ & $\begin{array}{l}\text { Wireless } \\
\text { communication with } \\
\text { a car } \\
\text { Bracelet needs } \\
\text { battery } \\
\text { Customers reception } \\
\text { is unknown }\end{array}$ \\
\hline
\end{tabular}

The first area for extension of those systems is accessibility for an average driver. Similarly, at the beginning touch screens were available only for the finest phone models. Now they are mounted even in low cost models. Today 3D gesture systems technology is somewhere between in the popularization route - It is available in high-mid-level class car VW Golf, but still majority of car brands do not offer this technology. Spreading this technology requires further research that would put the pressure on cost efficiency. Looking back, we can predict that it will happen and number of cars with $3 \mathrm{D}$ system recognition control will extend in the next few years.

Another important aspect that shall be improved soon is a number of available functional gestures. This corresponds to increasing complexity of a car multimedia environment, that needs higher number of gestures. More possible gestures mean easier and faster access to a specific function in complex car systems. In the article I mentioned a few examples, that detected more than simple swipes. It shows that detecting those type of gestures is possible, but application in cars needs further research and development in this area.

\section{References}

(1) W Cunningham: BMW finally embraces touchscreens, adds gesture control, https://www.cnet.com/roadshow/news/bmwfinally-embraces-touchscreens-adds-gesture-control/, accessed June 30, 2021.

(2) S. Akyol, U. Canzler, K. Bengler, W. Hahn: Gesture Control for use in Automobiles, IAPR Workshop on Machine Vision Applications, The University of Tokyo, Japan ,Nov. 28-30 2000.

(3) S. Zeiss, A. Marinc, A. Braun, T. Grosse-Puppendahl,S. Beck: A Gesture-Based Door Control Using Capacitive
Sensors, Distributed, Ambient, and Pervasive Interactions, 207-216, 2014, doi: 10.1007/978-3-319-07788-8_20.

(4) S .Frank, A. Kuijper: Enabling Driver Feet Gestures Using Capacitive Proximity Sensing, 14th International Conference on Intelligent Environments (IE), Rome, Italy, 2018, doi: 10.1109/IE.2018.00012.

(5) Microchip GestIC Technology home page, https://www.microchip.com/design-centers/capacitive-touchsensing/gestic-technology, accessed June 30, 2020.

(6) 2018 Jaguar XF Sportbrake gesture blind. https://www.youtube.com/watch?v=vPYzoB6viqU; accessed, June 30, 2020.

(7) H. Wei, P. Li, K. Tang, W. Wang, X. Chen: Alternating Electric Field-Based Static Gesture-Recognition Technology, Sensors 19(10), 2375, 2019, doi: 10.3390/s19102375.

(8) P.J.S. Leite: Automotive Gestures Recognition Based on Capacitive Sensing. University of Minho, Portugal, January 2017.

(9) G. Batchuluun, B. Odgerel, C.H. Lee: Hand Gesture Recognition Using an Infrared Proximity Sensor Array, The International Journal of Fuzzy Logic and Intelligent Systems 15(3), 185-191, Korea, doi: 10.5391/IJFIS.2015.15.3.186.

(10) H. Miura,H. Ishiwata,Y. Iida, Y. Matunaga, S. Numazaki, A. Morisita, N. Umeki, M. Doi: A 100Frame/s CMOS Active Pixel Sensor for 3D-Gesture Recognition System, 1999 IEEE International Solid-state Circuits Conference, San Francisco, USA, Feb. 1992.

(11) Gesture Recognition, Proximity Sensors Drive Advances in Automotive Infotainment, https://www.avnet.com/wps/portal/us/resources/article/gestur e-recognition-proximity-sensors-drive-advances-autoinfotainment/, accessed Jun. 30, 2021.

(12) A. Stoklosa: We Swipe Right on Volkswagen's ProductionBound Gesture-Control Interface. https://www.caranddriver.com/news/a15349506/we-swiperight-on-volkswagens-production-bound-gesture-controlinterface/, accessed Jun. 30, 2021.

(13) VW US Media Site, CES 2015: Volkswagen introduces advanced gesture control and networking for a new age of mobility, https://media.vw.com/en-us/releases/421, accessed Jun. 30, 2021.

(14) Volkswagen, Gesture Control in the Golf Volkswagen. https://www.youtube.com/watch? $\mathrm{v}=\mathrm{MD} 2 \mathrm{jNrJnPtA}$, accessed Jun.30, 2021

(15) Texas Instruments, mmWave Sensor Homepage, https://training.ti.com/60-ghz-mmwave-sensor-gesturerecognition, accessed Jun 30, 2020.

(16) Google, Soli Homepage, https://atap.google.com/soli/, accessed Jun. 30, 2021

(17) K.A. Smith,C. Csech,D. Murdoch,G. Shaker: Gesture Recognition Using mm-Wave Sensor for Human-Car Interface, IEEE Sensors Letters 2(2), 1-4, 2018, doi : 10.1109/LSENS.2018.2810093.

(18) S. Wang,J. Song,J. Lien,I. Poupyrev, O. Hilliges: Interacting with Soli: Exploring Fine-Grained Dynamic Gesture Recognition in the Radio-Frequency Spectrum, UIST '16: Proceedings of the 29th Annual Symposium on User Interface Software, Tokyo, Japan, Oct. 2016, doi: 10.1145/2984511.2984565.

(19) Y. Qifan,T. Hao,Z. Xuebing, L. Yin, Z. Sanfeng; Dolphin: Ultrasonic-Based Gesture Recognition on Smartphone Platform, 2014 IEEE 17th International Conference on Computational Science and Engineering. Chengdu, China, 2014, doi: 10.1109/CSE.2014.273.

(20) Y. Sang, L Shi,Y. Liu: Micro Hand Gesture Recognition System Using Ultrasonic Active Sensing, IEEE Access, 2018, doi: 10.1109/ACCESS.2018.2868268. 
(21) N. Uzzaman et al: A Comparative Analysis between Light Dependent and Ultrasonic Method of Gesture Recognition, 2016 3rd International Conference on Electrical Engineering and Information and Communication Technology, ICEEiCT, Sep. 2016, doi:10.1109/CEEICT.2016.7873101.

(22) M. Haid, B. Budaker, M. Geiger, D. Husfeldt, M. Hartmann, N.Berezowski: Inertial-Based Gesture Recognition for Artificial Intelligent Cockpit Control using Hidden Markov Models, 2019 IEEE International Conference on Consumer Electronics (ICCE), Las Vegas, USA. Jan. 11-13, 2019, doi: 10.1109/ICCE.2019.8662036.

(23) S. Oniga, J. Vegh, I. Orha: Intelligent human-machine interface using hand gestures recognition, Proceedings of 2012 IEEE International Conference on Automation, Quality and Testing, Robotics, Cluj-Napoca, Romania, 2012, doi: 10.1109/AQTR.2012.6237773.

(24) Y. Zhang, B. Liu, Z. Liu, J. Huang, R. Sun: Hand Gesture Classification with two-array Wrist-Mounted pressure sensors, 2019 IEEE 16th International Conference on Wearable and Implantable Body Sensor Networks (BSN). Chicago, USA, 2019, doi: 10.1109/BSN.2019.8771036. 